\title{
Characterization of Pathologic Cries of Newborns Based on Fundamental Frequency Estimation
}

\author{
Yasmina Kheddache, Chakib Tadj \\ Electrical Engineering Department, École de technologie Supérieure, Montréal, Canada \\ Email: Yasmina.kheddache.1@ens.etsmtl.ca, Chakib.Tadj@etsmtl.ca
}

Received June 2013

\begin{abstract}
The majority of the sick babies seem in good health at birth and early diagnosis of the various pathologies that can afflict newborn is crucial. For this reason we introduce in this paper the use of the fundamental frequency and the different modes of cries as Hyper-phonation, phonation and high pitched cries to differentiate the pathological cries from the cries of the healthy babies. The automatic estimation of these characteristics enabled us to establish a quantitative characterization of healthy newborn cries and pathologic newborn cries. The results obtained agree with the spectrographic analysis carried out on the healthy and pathologic cries of the newborns.
\end{abstract}

Keywords: Newborns’ Cry; Fundamental Frequency; Cry Modes

\section{Introduction}

For many researchers, the fundamental frequency F0 which represents the speed of opening and closure of the vocal folds [1] is considered as the most important in the perception of the infant's cries by adults. Generally the increase of F0 is associated with a negative perception of the cries. The cries with a high F0 are valued as aversive, sign of emergency, distress and disease [2].

Cry analysis can be an additional tool in the clinical diagnosis of newborn. The fundamental frequency of newborn cry has been found to be a discriminative characteristic in several medical problems [1].

Cry analysis presented in this paper falls within the research on the relationship between the disease and the characteristics of the cry. Newborns do not have phonatory control due to neurological immaturity at early age [3]. The main objective of this work is to analyze cries of healthy and newborn with different categories of diseases in order to evaluate a fundamental frequency of these cries. We also establish quantitative relationships between the different modes of cries and studied pathology.

The SIFT algorithm 'Simple Inverse Filtering Tracking' is used for estimation of the fundamental frequency because the performance of this algorithm has been tested on a real database of cries by $[4,5]$.

This paper is organized as follows: Section 2 presents definitions of fundamental frequency of cries. Section 3 presents previous works. Section 4 presents our adopted methodology for automatic estimation of F0, phonic, hyper-phonic and high pitched segments for healthy and pathological cries. Section 5 presents the database used in this study. The next sections present the results obtained and we conclude this paper with a conclusion.

\section{Fundamental Frequency of Cries}

Fundamental frequency (F0) is the average vibratory frequency (in $\mathrm{Hz}$ ) of the vocal folds. The sound spectrogram displays the voiced tone as black lines. The fundamental frequency is the lowest line and its harmonics appear above it as parallel lines. The maximal fundamental frequency corresponds to the highest measurable point of the fundamental frequency and the minimal fundamental frequency corresponds to the lowest measurable point of the fundamental frequency on the spectrogram [6].

Lester et al. [2] defines three identifiable modes of cries due to vocal cord vibrations: 1) Basic cry or phonation with F0 350 - 750 Hz; 2) Cry with high fundamental frequency F0 (750 - $1000 \mathrm{~Hz})$ or Hyper-phonation F0 (1000 - $2000 \mathrm{~Hz})$ and 3) Noisy, turbulent or dysphonic cries.

\section{Previous Works}

Several authors focused their investigations on the healthy children since birthday to one year age. Their work is based on observation and spectrographic analysis of healthy and pathological cries signals of newborns. They found that there is no significant difference between the preterm newborn cries and the full-term newborn cries. They also found that there is not variation of 
F0 compared to the sex and the gestational age [3]. In the case of the newborn with various pathologies, it was shown that the level of the cries is high and presents a weak punctuation, high irritability and low physiological stability $[1,3]$.The authors in this research area show that the cries of healthy full-term newborn are characterized by an F0 varying between 400 and $600 \mathrm{~Hz}$ and averaging $450 \mathrm{~Hz}[5,7]$. The cries of healthy premature newborns can be more intense or similar to the cries of full-term newborns [1]. The cries of newborns with neurological disturbances exhibit auditory abnormalities [6]. Hyperphonic cries and very high-pitched cries are associated with neurological problems [2] and neonatal risk [8].

\section{Methodology}

Our methodology for the characterization of healthy and pathologic cries based on F0 estimation is represented on the simplified diagram blocks illustrated in Figure 1.

The following approach was taken when estimating fundamental frequency and the different frequency modes of cries:

- Recording of healthy and pathologic cries of newborns.

- Noise filtering and segmenting recordings into useful and non-useful portions.

- Estimation of F0 in short intervals typically of $20 \mathrm{~ms}$ interlaced frame with $10 \mathrm{~ms}$ recovering using SIFT algorithm (Simple Inverse Filtering Tracking) [4,9]. The main steps of this algorithm are presented in Figure 2.

- Estimating of phonic and hyper-phonic segments, as well as segments with high F0 as defined in Table 1 for all cries studied.

- Identification of relationships between cries characteristics and studied pathologies.

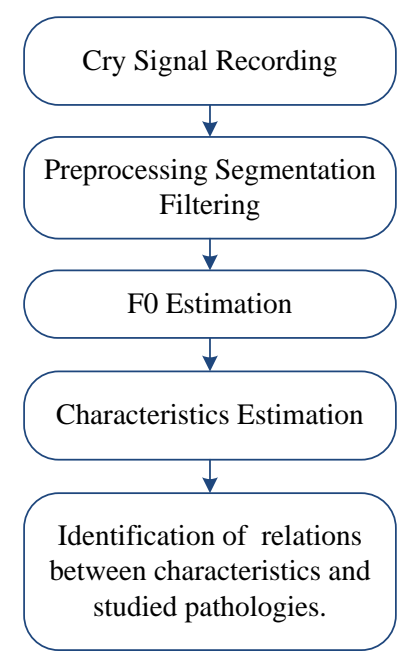

Figure 1. Newborn cries characterization based on F0 estimation.

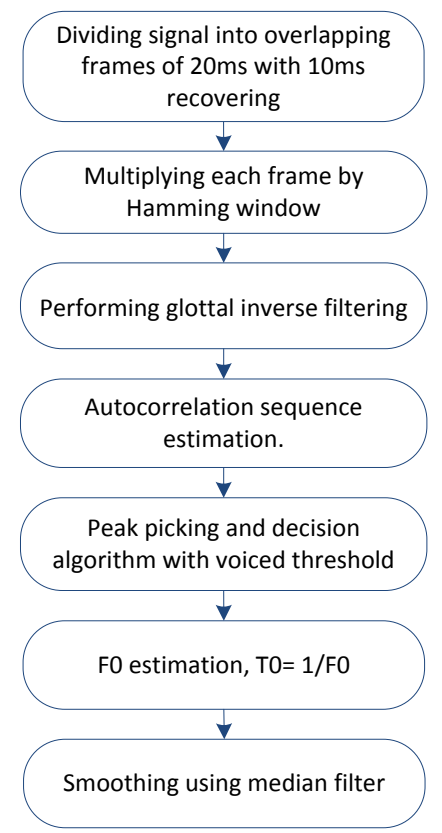

Figure 2. The main steps of SIFT algorithm.

Table 1. Measured characteristics of cries.

\begin{tabular}{cc}
\hline Characteristics & Definition \\
\hline Hyper-phonation & $\begin{array}{c}\text { The average percentage of } 20 \mathrm{~ms} \text { blocks } \\
\text { having a F0 }>1000 \mathrm{~Hz} .\end{array}$ \\
Phonation & $\begin{array}{c}\text { The average percentage of } 20 \mathrm{~ms} \text { blocks } \\
\text { having a F0 }<750 \mathrm{~Hz}\end{array}$ \\
High-pitched & $\begin{array}{l}\text { The average percentage of } 20 \mathrm{~ms} \text { blocks } \\
\text { having a F0 in the } 750-1000 \mathrm{~Hz} \text { range }\end{array}$ \\
\hline
\end{tabular}

\section{Database: Cry Recording}

The database used contains 2800 cry samples of 1s duration from 48 of newborn babies. 1774 from healthy newborn (among them 764 are premature) and 1010 from newborn who present some diseases (among them 628 are premature) as shown in Table 2.

These cries were collected with the aid of medical collaborators of neonatology department at Saint-Justine Hospital in Montreal. They concern full-term and preterm newborn aged 1 to 30 days. The conditions in which the cries are registered are: hunger, sampling blood, change of diapers. The recording of cries is done using a small recorder, at a distance of $10 \mathrm{~cm}$ of babies' mouth with a sampling rate of $44.1 \mathrm{kHz}$.

For each baby, three recordings of duration 2 to $3 \mathrm{mi}-$ nutes are made with at least one hour interval after each recording session (over a period of ten days at most). The time, date and gender, date of birth, diagnosis, and reason of cries are noted for each episode of cry.

\section{Results and Analysis}

We developed our own measuring tool using Matlab. 
Table 2. Pathologies studied.

\begin{tabular}{llc}
\hline \multicolumn{1}{c}{ Category } & \multicolumn{1}{c}{ Pathology } & Sample size \\
\hline \multirow{2}{*}{ Full-term } & Healthy & 1010 \\
newborn (t) & Thrombosis in the vena cava & 77 \\
& Meningitis & 115 \\
& Asphyxia & 190 \\
& Healthy & 764 \\
Preterm & IUGR- Microcephaly & 78 \\
newborn (P) & (Intra-Uterine Growth Retardation) & 280 \\
& Gastroschisis & 270 \\
\hline
\end{tabular}

This tool estimates F0 as well as the average percentages of phonic, hyper-phonic and high pitched segments. Examples of spectrograms and F0 estimation are reported in Figure 3 for two pathologic cries (asphyxia and gastroschisis) and also for a healthy newborn. Notice high F0 and more irregular spectrograms and F0 for pathologic cries compared to healthy cry. The range of F0 for pathologic cries is $150 \mathrm{~Hz}-1600 \mathrm{~Hz}$ and for healthy cry around $450 \mathrm{~Hz}$. The estimated values of frequency characteristics listed in Table $\mathbf{1}$ for healthy and pathologic newborn's cry are presented in Figures $\mathbf{4}$ and $\mathbf{5}$ according to pathologies studied and gestational age of babies.

The results from this study show in Figure 4 that the cries of healthy full-term or preterm newborns are rather phonics with a presence of $89 \%$ of segments with a F0 < $750 \mathrm{HZ}$ and round 9\% of segments with high F0 > 750 Hz. Figure 5 shows that the cries of healthy full-term or preterm newborns contain round 6\% of Hyper-phonic segment. On the other hand, the cries of the sick newborns contain more pitched and hyper-phonic segment compared to the healthy newborns. According to the pathologies studied, the percentage varied from $11 \%$ to $28 \%$ of segments with a F0 $>750 \mathrm{~Hz}$. These percentages vary from one pathological condition to another, and are dependant to level of prematurity.

We notice in Figures $\mathbf{4}$ and 5, which the cries of newborns with asphyxia contain $28 \%$ of high pitched segments (F0 > $750 \mathrm{~Hz}$ ) among them 25\% are hyperphonic. In the case of meningitis, the cries contain respectively $11 \%$ and $8 \%$ of high pitched segments (F0 $>750 \mathrm{~Hz}$ ) and hyper-phonic segments.

The cries of newborns with microcephaly associated with IUGR present $25 \%$ of high pitched segments (F0 > $750 \mathrm{~Hz}$ ). These cries are more hyper-phonic with $17 \%$ of segment with F0 > $1000 \mathrm{~Hz}$. The cries of newborns with congenital malformation gastroschisis contain $13 \%$ of high pitched segments with F0 > $750 \mathrm{~Hz}$. The percentage of hyperphonation is $10 \%$ of segment with F0 $>1000 \mathrm{~Hz}$.

The cries of newborns with heart defect (Thrombosis

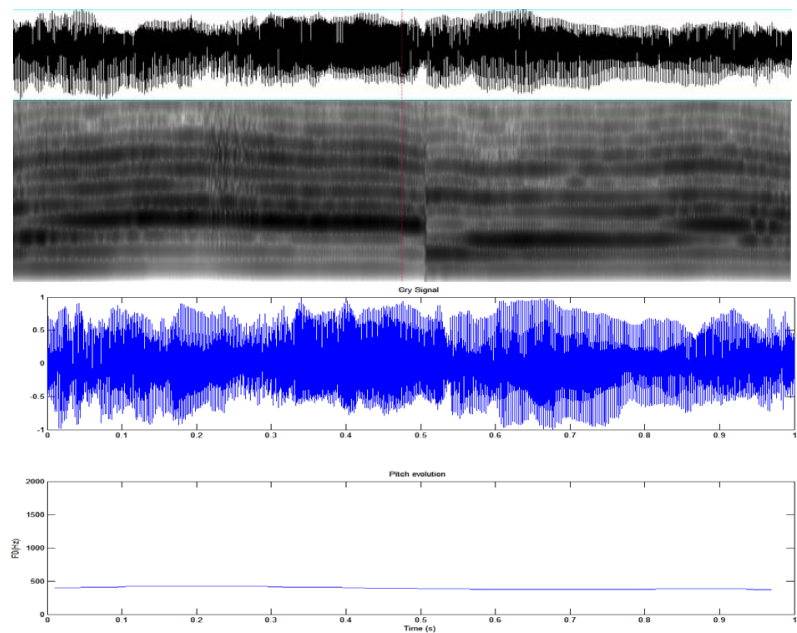

(a)
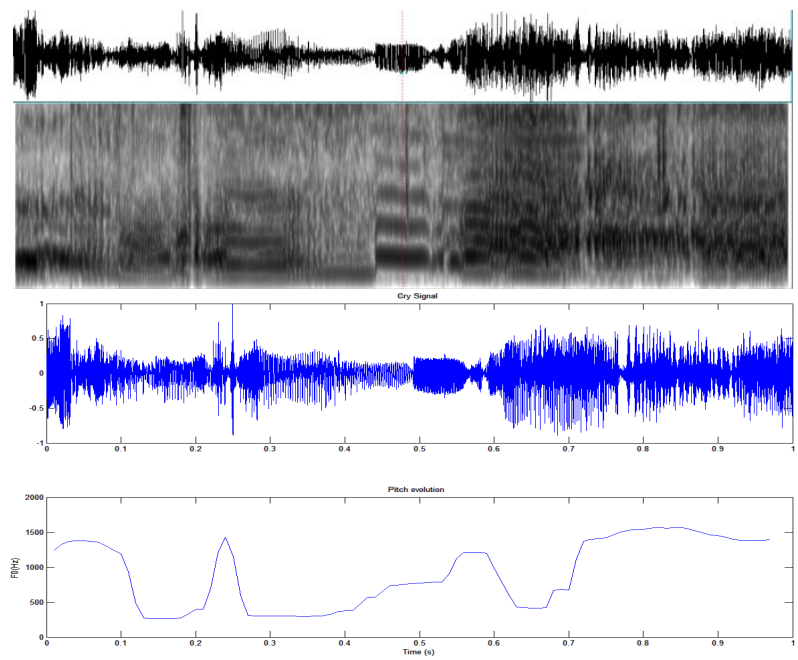

(b)
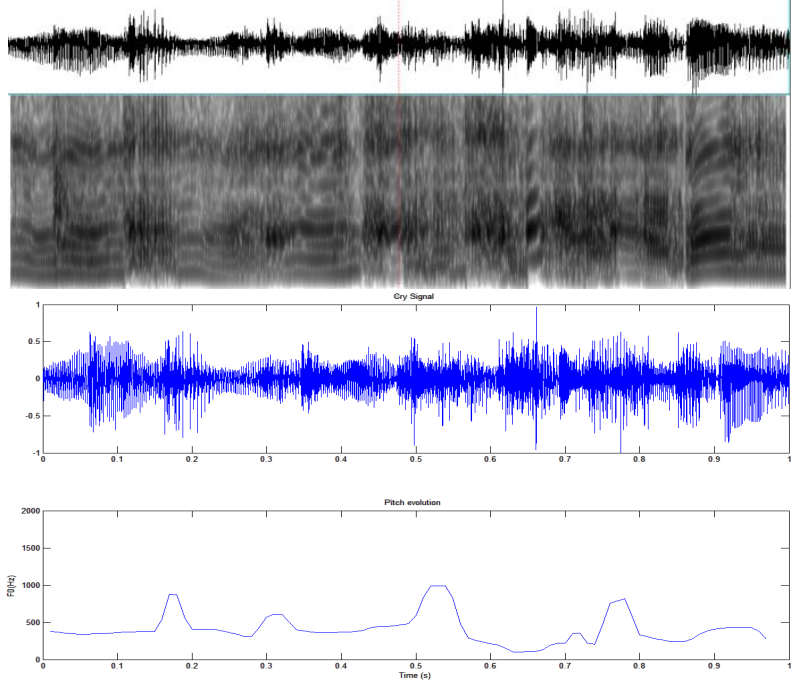

(c)

Figure 3. Spectrograms and Fo estimation for healthy and pathologic cries. (a) Full-Term healthy baby; (b) Full-Term baby with Asphyxia; (c) Premature baby with gastroschisis. 


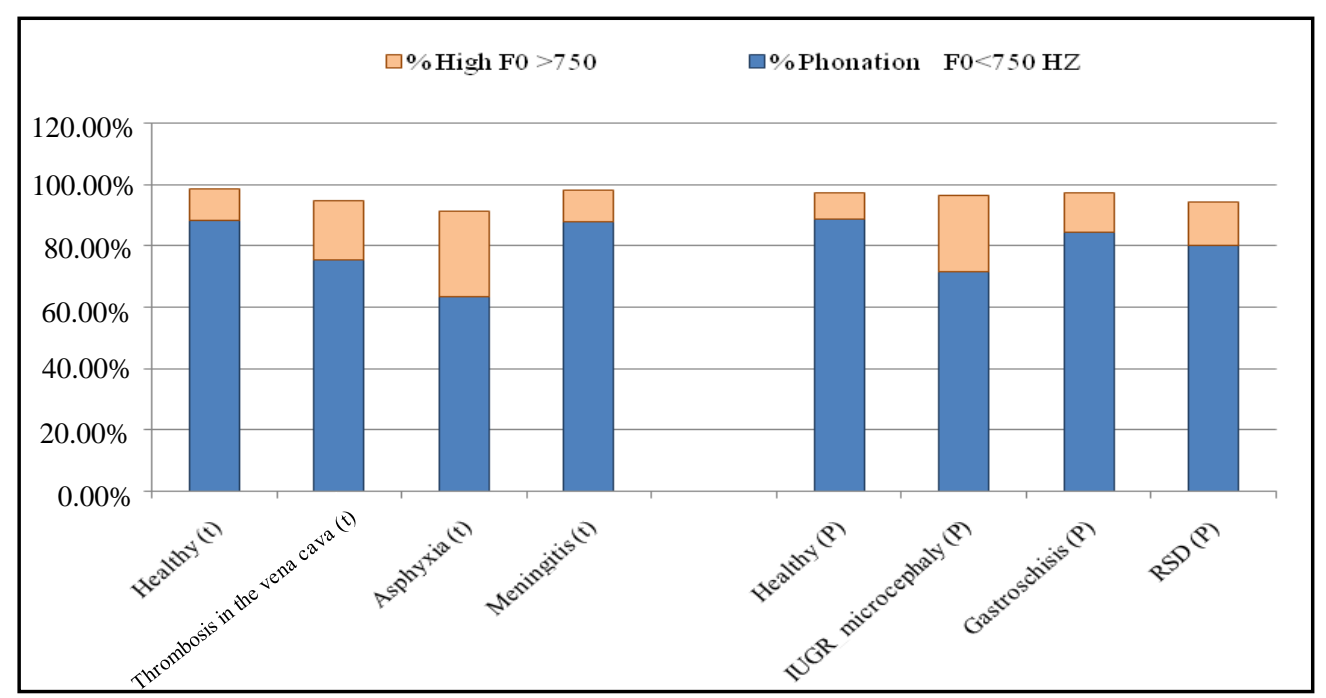

Figure 4. The average percentage of phonic and high pitched segments (F0 $>750 \mathrm{~Hz})$ by pathologies.

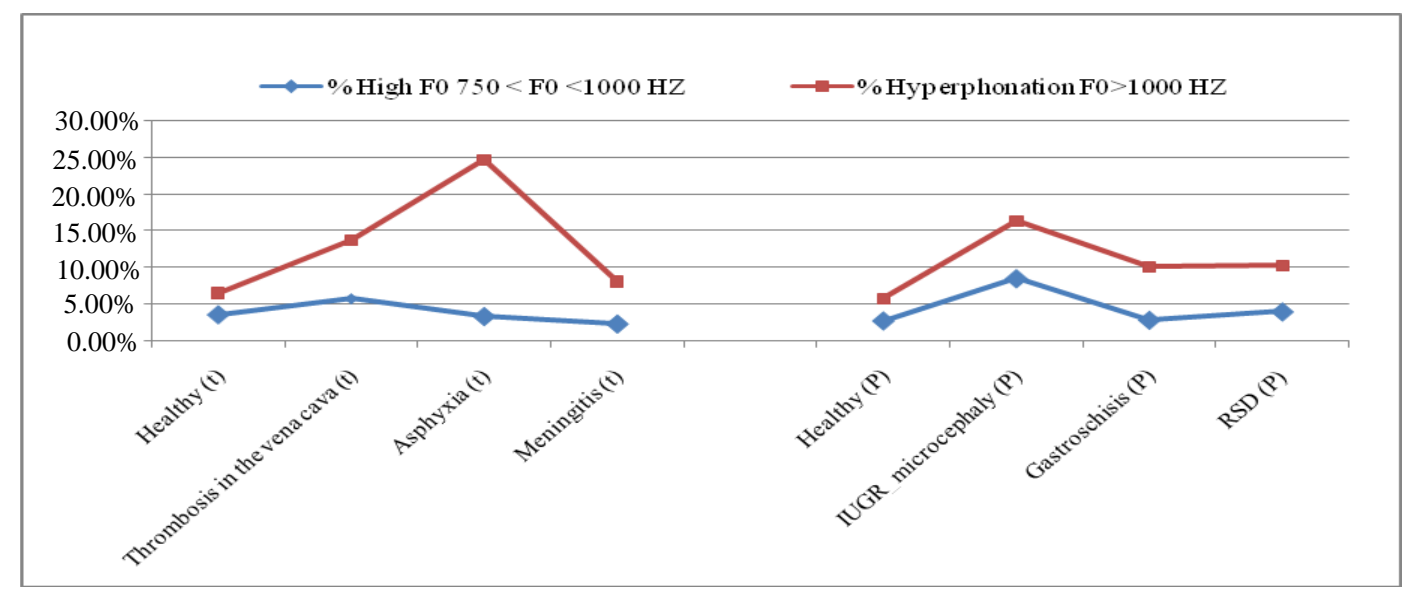

Figure 5. The average percentage of hyper-phonic $(\mathrm{FO}>1000 \mathrm{~Hz})$ and high pitched segments $(750<$ F0 $<1000 \mathrm{~Hz})$ by pathologies.

in the vena cava), include $20 \%$ of segments with F0 > $750 \mathrm{~Hz}$, among them 14\% are hyper-phonic. The cries of newborn with RDS (Respiratory Distress Syndrome) include $15 \%$ of segments with F0 $>750 \mathrm{~Hz}$ among them $11 \%$ are hyper-phonic.

The highest average percentage of hyper-phonic cries is found in the category of neurological problems (asphyxia, microcephaly-IUGR) and also the highest average percentage of high pitched cries with $750<\mathrm{F} 0<1000 \mathrm{~Hz}$ is found in the category of neurological problems (microcephaly-IUGR).

\section{Conclusions}

This study mostly deals with characterization of healthy and pathologic newborn's cry according to the modes of cry as hyper-phonation, phonation, and high-pitched cry.

The conclusion from this work is that there are clear differences in frequency characteristics of healthy and pathologic cries. This difference can be used in pediatric diagnosis. The results obtained are very encouraging because they are consistent with spectrographic studies of crying newborns [1,2,10].

This work is still in progress. The results obtained by using different modes of cry as discriminative characteristics for healthy and pathologic newborn's cry can be improved using other acoustic characteristics cited in the literature in association with severe medical conditions. More significant results can be expected using the developed tool on a larger database with a greater variety of pathologies and more subjects for each pathology.

\section{Acknowledgements}

We would like to thank Dr. Barrington and members of neonatology group of Saint-Justine Hospital in Montreal (QC) for their dedication of the collection of the Infant's cry database. This research work has been funded by a 
grant from the Bill \& Melinda Gates Foundation through the Grand Challenges Explorations Initiative.

\section{REFERENCES}

[1] B. M. Lester and L. L. LaGasse, "Crying," Elsevier, Amsterdam, 2008, pp. 80-90.

[2] L. L. LaGasse, A. R. Neal and B. M. Lester, “Assessment of Infant Cry: Acoustic Cry Analysis and Parental Perception," Mental Retardation and Developmental Disabilities Research Reviews, Vol. 11, No. 1, 2005, pp. 8393. http://dx.doi.org/10.1002/mrdd.20050

[3] A. Verduzco-Mendoza, E. Arch-Tirado, C. A. Garcia and J. L. Ibarra, "Qualitative and Quantitative Crying Analysis of New Born Babies Delivered Under High Risk Gestation,” Springer-Verlag Berlin Heidelberg, 2009, pp. 320327.

[4] D. Lederman, "Estimation of Infants’ Cry Fundamental Frequency Using a Modified SIFT Algotithm,” 2010. Eprint arXiv: 1009.2796

[5] C. Manfredi, L. Bocchi, S. Orlandi, L. Spaccaterra and G.
P. Donzelli, "High-Resolution Cry Analysis in Preterm Newborn Infants,” Elsevier, Amsterdam, 2009, pp. 528532.

[6] O. Wasz-Hockert, K. Michelsson and J. Lind "Twenty-Five Years of Scandinavian Cry Research,” Springer, New York, 1985, pp. 83-104.

[7] K. Michelson, K. Eklund, P. Leppanen and H. Lyytinen, "Cry Characteristics of 172 Healthy 1- to-7 Day Old Infants,” Folia Phoniatr Logopaedica, 2002, pp. 190-200.

[8] C. Manfredi, V. Tocchioni and L. Bocchi, "A Robust Tool for Newborn Infant Cry Analysis," Proceedings 28th IEEE EMBS Annul International Conference, 2006.

[9] J. D. Markel, "The SIFT Algorithm for Fundamental Frequency Estimation,” IEEE Transactions on Audio and Electroacoustics, Vol. 20, No. 5, 1972.

[10] K. Michelson, H. Todd de Barra and O. Michelson, "Sound Spectrographic Cry Analysis and Mothers Perception of Their Infant's Crying," Nova Science Publishers, New York, Chapter 2, 2007, pp. 31-64. 